\title{
Study of the Light Coupling Efficiency of OLEDs Using a Nanostructured Glass Substrate
}

\author{
Min Won Lee, ${ }^{1}$ Siegfried Chicot, ${ }^{1}$ Chii-Chang Chen, ${ }^{2}$ Mahmoud Chakaroun, \\ Getachew Ayenew, ${ }^{1}$ Alexis Fischer, ${ }^{1}$ and Azzedine Boudrioua ${ }^{1}$ \\ ${ }^{1}$ Laboratoire de Physique des Lasers, UMR CNRS 7538, Université Paris 13, Sorbonne Paris Cité, Villetaneuse, France \\ ${ }^{2}$ Department of Optics and Photonics, National Central University, Taiwan
}

Correspondence should be addressed to Min Won Lee; min.lee@univ-paris13.fr

Received 30 November 2013; Revised 20 February 2014; Accepted 21 February 2014; Published 24 March 2014

Academic Editor: Vincent Jousseaume

Copyright (C) 2014 Min Won Lee et al. This is an open access article distributed under the Creative Commons Attribution License, which permits unrestricted use, distribution, and reproduction in any medium, provided the original work is properly cited.

\begin{abstract}
We study theoretically the enhancement of the light extraction from an OLED (Organic Light-Emitting Diode) with nanoairbubbles embedded inside a glass substrate. Due to such a nanostructure inside the substrate, the critical angle which limits the light extraction outside the substrate from the OLED is increased. The theoretical results show that the nanoair bubbles near by the substrate surface can improve the efficiency of the light extraction by 7\%. Such a substrate may also be suitable for photovoltaic cells or display screens.
\end{abstract}

\section{Introduction}

Over the last decade, organic materials have attracted much attention due to their huge potential of applications such as OLED (Organic Light-Emitting Diode) and organic photovoltaic cells. As promising materials, the enhancement of their efficiency has widely been studied [1-4]. Although the internal quantum efficiency of OLEDs can be achieved near $100 \%$ [5-8], the light emitted by the OLED is largely wasted because of the total internal reflection inside the substrate. The most common substrate is glass substrate coated with an ITO (Indium Tin Oxide) layer. The ITO layer is used as a transparent anode electrode, but its refractive index is higher than that of organic materials. Therefore, much of light is trapped in the layer due to the internal reflection. Moreover, the light must again cross the glass-air boundary. This produces a total reflection of the light emitted from the organic materials beyond the critical angle. Therefore only small amount of light is coupled out of the ITO layer and glass substrate, and the rest is trapped inside the ITO layer and substrate as wave-guided modes. It has been reported that the external coupling efficiency of light from conventional OLEDs is only $20 \%$ [6-8]. A number of studies have been done to improve the coupling efficiency from OLEDs or photovoltaic cells such as addition of a diffusive layer on the substrate [9], nanomesh electrodes [10], nanoparticles [11], and microlens array $[12,13]$. Corrugated structure and a quasiperiodic buckling structure were also proposed with an excellent outcoupling efficiency [14, 15]. A low-index grid embedded in the organic layer and a monolayer of $\mathrm{SiO}_{2}$ microspheres are also suggested for this purpose $[7,16]$. Recently, patterning nanostructures on the substrate surface has been developed in order to increase the light extraction efficiency in [17]. This method may also be applied to OLEDs on glass substrate. However, as they are exposed to air directly, such structures are vulnerable to external shocks. So the structure can be destroyed or damaged if mechanical or chemical cleaning process of such substrates is applied, thus reducing the coupling efficiency.

In this work, we study numerically a nanostructure inside the glass substrate in order to enhance the coupling efficiency of OLEDs. The 2D air holes are positioned inside the substrate near the surface. The extraction efficiency of such a nanostructure in a glass substrate for the light emitted from OLED is studied numerically taking into account three main parameters: the period of air holes, the air hole diameter, and the depth of air holes from the substrate surface. The incident angle at which the light is escaped from the substrate 


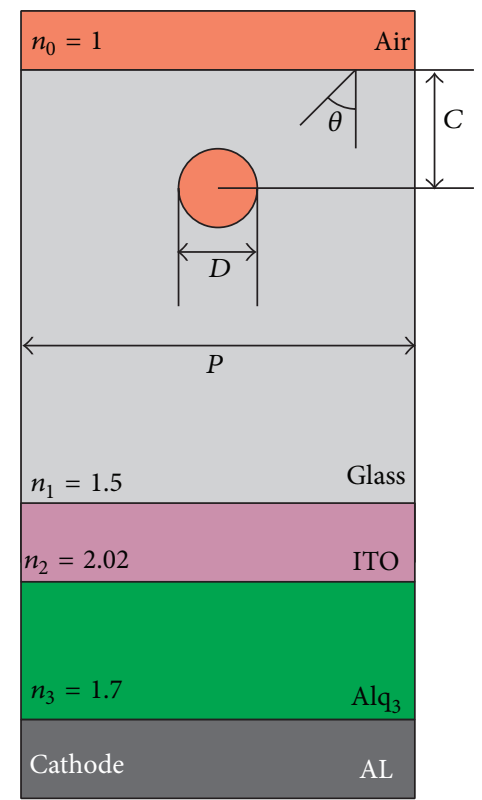

FIGURE 1: Schematic illustration of the OLED structure used in our simulation.

is also investigated. The nanostructure embedded in the glass substrate proposed in our work can avoid any damage or deterioration of the structure due to mechanical or chemical cleaning process as the nanoair bubbles are protected in the glass substrate.

\section{Nanostructure in Glass Substrate}

The structure of an OLED studied in our work is illustrated schematically in Figure 1. The organic layer is made of $\mathrm{Alq}_{3}$ (Tris(8-hydroxyquinolinato)aluminium) with a thickness of $120 \mathrm{~nm}$ and its refractive index is $n_{3}=1.7$. The ITO layer is found on the organic layer with a thickness of $100 \mathrm{~nm}$ and a refractive index of $n_{2}=2.02$. It is used to inject the current as the anode whilst the aluminium layer at the bottom ensures the current flow through the organic layer as the cathode. The glass substrate has an index of $n_{1}=1.5$ and a thickness of $2 \mathrm{~mm}$. The air holes with a unity refractive index are positioned near the substrate surface. The light emitted by the organic layer is collected in the air with a unity air index. In fact, the typical structure consists of hole transport and electron transport layers. But we have neglected these layers for the sake of simplicity in our simulation as they do not affect much the coupling efficiency.

In order to investigate the external coupling efficiency of the light emitted from the organic layer, three main parameters are taken into account in the simulation: the period of air holes $P$, the air hole diameter $D$, and the depth of air holes from the substrate surface $C$. In the simulation, $P>D$ is maintained to avoid overlaps between the air holes. $C>D / 2$ is also kept, so that the air holes remain inside the substrate. The angle $\theta$ is the incident angle of the light emitted from the organic layer with respect to the normal of the substrate surface.

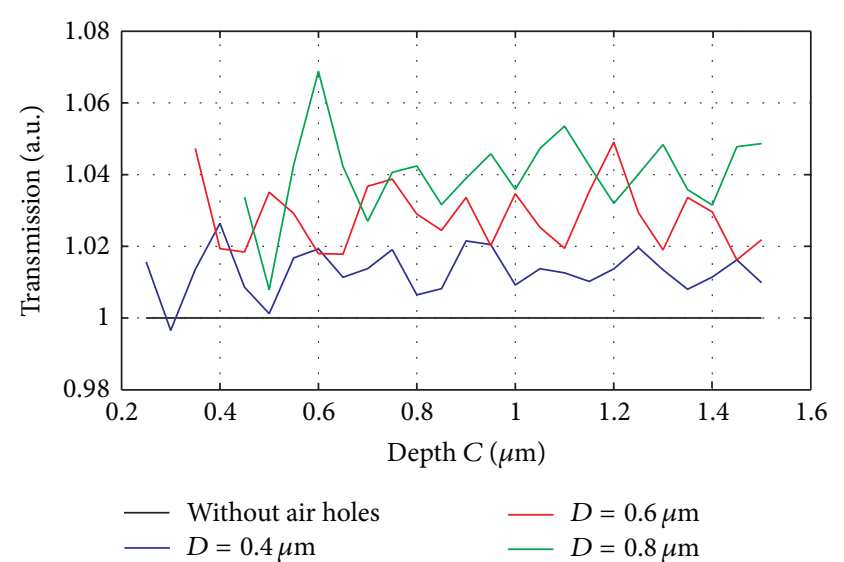

FIGURE 2: Light transmission as a function of the depth of air holes $C$ with a period of $P=2.5 \mu \mathrm{m}$.

We have adapted the RCWA (Rigorous Coupled Wave Analysis) method in 2 dimensions to our light coupling simulation $[18,19]$. The emission wavelength of $\mathrm{Alq}_{3}$ is known to be $520 \mathrm{~nm}$ at a thickness of $120 \mathrm{~nm}$ [20]. The FWHM (Full Width Half Maximum) of the spectrum is $50 \mathrm{~nm}$ and the broad spectrum is integrated to calculate the OLED output intensity. The light transmissions of the TE and TM modes are all summed up on the incident angles between $0^{\circ} \leq \theta \leq$ $89^{\circ}$. The extraction efficiency is investigated from the light transmissions with and without the air hole structure.

\section{Numerical Results}

Figure 2 shows the light transmission as a function of the depth of air holes $C$ with 3 different diameters $D$ and a period of $P=2.5 \mu \mathrm{m}$. The black straight line is the case without the nanostructure. This case is used as a reference with a unity transmission value in our work. From the figure, the maximum light extraction is obtained as 1.07 at $C=0.6 \mu \mathrm{m}$ and $D=0.8 \mu \mathrm{m}$. It is clear that the nanostructure with these parameters allows increasing the light coupling by $7 \%$. Other periods are also investigated in the simulation, but the light extraction is a bit lower than that with $P=2.5 \mu \mathrm{m}$. These parameters should be used in order to fabricate the nanostructure in the glass substrate.

These parameters are also used to calculate the light transmission from the organic layer as a function of the incident angle $\theta$. In Figure 3, the lights extracted from the OLED are shown with and without the nanostructure. The black curve represents the light extracted from the bulk substrate and the red curve represents the nanostructured substrate. In the case of bulk substrate, the light is transmitted up to $41^{\circ}$ which is the critical angle $\theta_{c}$ for extracting light from the organic layer through the ITO layer. Actually, this angle corresponds to the one calculated by Snell-Descartes Law in such a configuration with three refractive indexes $\left(n_{1}=1.5\right.$, $n_{2}=2.02$, and $\left.n_{3}=1.7\right)$. For $>\theta_{c}$, the light is trapped in both the ITO layer and substrate as wave-guided modes. On the other hand, in the case of nanostructured substrate, $50 \%$ of the light is transmitted through the substrate until $61^{\circ}$. 


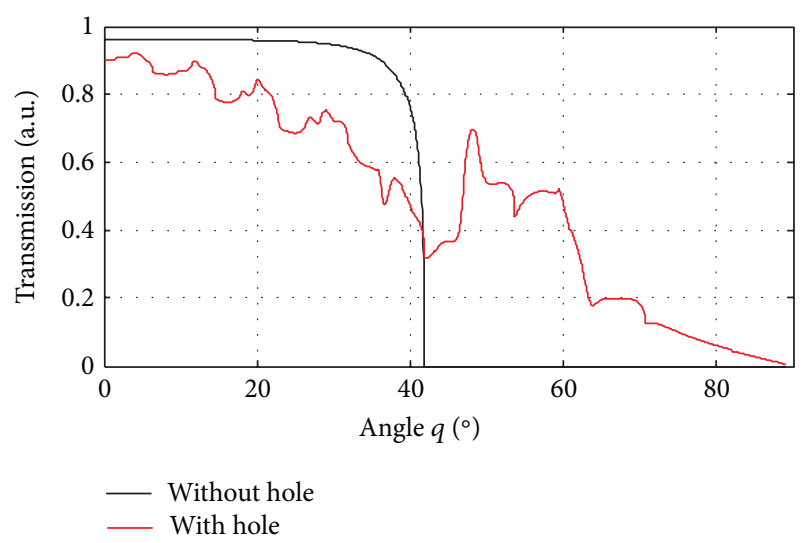

FIgURE 3: Light transmission as a function of the incident angle $\theta$.

A small amount of light can still be extracted up to $88^{\circ}$ whilst the light is trapped beyond $41^{\circ}$ in the bulk case. Hence, the critical angle is increased to $88^{\circ}$ which is twice greater than that of the bulk substrate case and the overall light intensity is gained due to the wide extraction angle from the nanostructured substrate. It is clearly seen that the nanostructure allows increasing the critical angle to extract more light. This is the promising results for the next generation of OLEDs and photovoltaic cells.

\section{Conclusions}

In this work, we have studied theoretically the improvement of the light coupling efficiency using air hole nanostructure inside a glass substrate. In the simulation, we have taken into account three parameters: the air hole period, the air hole diameter, and the depth of the air hole from the substrate surface. Our results show that the efficiency of light extraction increases by $7 \%$ due to the air hole nanostructure at specific parameters. Besides, the light emitted from the organic layer is extracted through the ITO glass up to an incident angle of $88^{\circ}$ which is twice higher than that without nanostructure $\left(41^{\circ}\right)$. In fact, the wide angle allows higher efficiency of light extraction. Such an embedded nanostructure may improve the efficiency of photovoltaic cells or the quality of display screens. Moreover, it would not suffer much structure deterioration in case of chemical or mechanical cleaning process.

In order to realize such a structure, two techniques may be proposed. Femtosecond lasers have been used to create microchannels with a cross-sectional size of around $1 \mu \mathrm{m}$ in glass [21]. Higher numerical aperture objectives of femtosecond lasers may be used to fabricate microchannels with a nanometer size. This technique would require only a femtosecond laser and some accessory components such as translation stages and femto-second lasers are now readily available in the market. Therefore, it would not require heavy process. Our first attempts on glass and sapphire show that laser ablation with an $193 \mathrm{~nm}$ nanosecond excimer laser is a promising technique for etching microstructures. Photolithography process could also be an alternative method. Microchannels can be etched on the surface of glass substrate.
Then the surface can be covered by a glass membrane by using wafer bonding technique [22]. The nanostructure could be embedded in glass by using these techniques to enhance device performance. As this structure offers a wide angle of light extraction, it would be suited to wide angle applications.

\section{Conflict of Interests}

The authors declare that there is no conflict of interests regarding the publication of this paper.

\section{Acknowledgment}

The author Siegfried Chicot would like to thank the Taiwanese Embassy for his scholarship which allows him to carry out this work in Taiwan for an M.S. degree.

\section{References}

[1] N. K. Patel, S. Cinà, and J. H. Burroughes, "High-efficiency organic light-emitting diodes," IEEE Journal on Selected Topics in Quantum Electronics, vol. 8, no. 2, pp. 346-361, 2002.

[2] Y. G. Lee, S. K. Kang, T. S. Oh, H.-N. Lee, S. Lee, and K. H. Koh, "Comparison of two cohost systems for doped red organic lightemitting devices in an effort to improve the efficiency and the lifetime," Organic Electronics, vol. 9, no. 3, pp. 339-346, 2008.

[3] Z. B. Wang, M. G. Helander, J. Qiu et al., "Unlocking the full potential of organic light-emitting diodes on flexible plastic," Nature Photonics, vol. 5, no. 12, pp. 753-757, 2011.

[4] T. H. Han, Y. Lee, M. R. Choi et al., "Extremely efficient flexible organic light-emitting diodes with modified graphene anode," Nature Photonics, vol. 6, no. 2, pp. 105-110, 2012.

[5] C. Adachi, M. A. Baldo, M. E. Thompson, and S. R. Forrest, "Nearly $100 \%$ internal phosphorescence efficiency in an organic light emitting device," Journal of Applied Physics, vol. 90, no. 10, pp. 5048-5051, 2001.

[6] S. R. Forrest, "The road to high efficiency organic light emitting devices," Organic Electronics, vol. 4, no. 2-3, pp. 45-48, 2003.

[7] Y. Sun and S. R. Forrest, "Enhanced light out-coupling of organic light-emitting devices using embedded low-index grids," Nature Photonics, vol. 2, no. 8, pp. 483-487, 2008.

[8] K. Saxena, V. K. Jain, and D. S. Mehta, "A review on the light extraction techniques in organic electroluminescent devices," Optical Materials, vol. 32, no. 1, pp. 221-233, 2009.

[9] T. Nakamura, N. Tsutsumi, N. Juni, and H. Fujii, "Improvement of coupling-out efficiency in organic electroluminescent devices by addition of a diffusive layer," Journal of Applied Physics, vol. 96, no. 11, pp. 6016-6022, 2004.

[10] Y. H. Ho, K. Y. Chen, K. Y. Peng, M. C. Tsai, W. C. Tian, and P. $\mathrm{K}$. Wei, "Enhanced light out-coupling of organic light-emitting diode using metallic nanomesh electrodes and microlens array," Optics Express, vol. 21, no. 7, pp. 8535-8543, 2013.

[11] S. Y. Hsu, M. C. Lee, K. L. Lee, and P. K. Wei, "Extraction enhancement in organic light emitting devices by using metallic nanowire arrays," Applied Physics Letters, vol. 92, no. 1, Article ID 013303, 2008.

[12] F. Galeotti, W. Mrz, G. Scavia, and C. Botta, "Microlens arrays for light extraction enhancement in organic light-emitting diodes: a facile approach," Organic Electronics, vol. 14, no. 1, pp. 212-218, 2013. 
[13] J. Wang, W. Li, and C. Wang, "Improving light outcoupling efficiency for OLEDs with microlens array fabricated on transparent substrate," Journal of Nanomaterials, vol. 2014, Article ID 289752, 6 pages, 2014.

[14] W. J. Hyun, S. H. Im, O. O. Park, and B. D. Chin, "Corrugated structure through a spin-coating process for enhanced light extraction from organic light-emitting diodes," Organic Electronics, vol. 13, no. 4, pp. 579-585, 2012.

[15] W. H. Koo, S. M. Jeong, F. Araoka et al., "Light extraction from organic light-emitting diodes enhanced by spontaneously formed buckles," Nature Photonics, vol. 4, no. 4, pp. 222-226, 2010.

[16] T. Bocksrocker, J. Hoffmann, C. Eschenbaum et al., "Microspherically textured organic light emitting diodes: a simple way towards highly increased light extraction," Organic Electronics, vol. 14, no. 1, pp. 396-401, 2013.

[17] W. Cai and R. Piestun, "Patterning of silica microsphere monolayers with focused femtosecond laser pulses," Applied Physics Letters, vol. 88, no. 11, Article ID 111112, 2006.

[18] M. G. Moharam, D. A. Pommet, E. B. Grann, and T. K. Gaylord, "Stable implementation of the rigorous coupled-wave analysis for surface-relief gratings: enhanced transmittance matrix approach," Journal of the Optical Society of America A, vol. 12, no. 5, pp. 1077-1086, 1995.

[19] M. G. M. M. Kraaij and J. M. L. Maubach, "A more efficient rigorous coupled-wave analysis algorithm," in Progress in Industrial Mathematics at ECMI, 2004, A. Bucchianico, R. M. M. Mattheij, and M. A. Peletier, Eds., vol. 8, pp. 164-168, Springer, Berlin, Germany, 2004.

[20] V. Bulović, V. B. Khalfin, G. Gu, P. E. Burrows, D. Z. Garbuzov, and S. R. Forrest, "Weak microcavity effects in organic lightemitting devices," Physical Review B-Condensed Matter and Materials Physics, vol. 58, no. 7, pp. 3730-3740, 1998.

[21] C. Hnatovsky, R. S. Taylor, E. Simova et al., "Fabrication of microchannels in glass using focused femtosecond laser radiation and selective chemical etching," Applied Physics A: Materials Science and Processing, vol. 84, no. 1-2, pp. 47-61, 2006.

[22] T. Rogers and N. Aitken, "Wafer bonding processes for the manufacture of microsystems," in Proceedings of the ASME 2nd International Conference on Integration and Commerciauzation of Micro and Nanosystems (MicroNano '08), pp. 705-710, June 2008. 

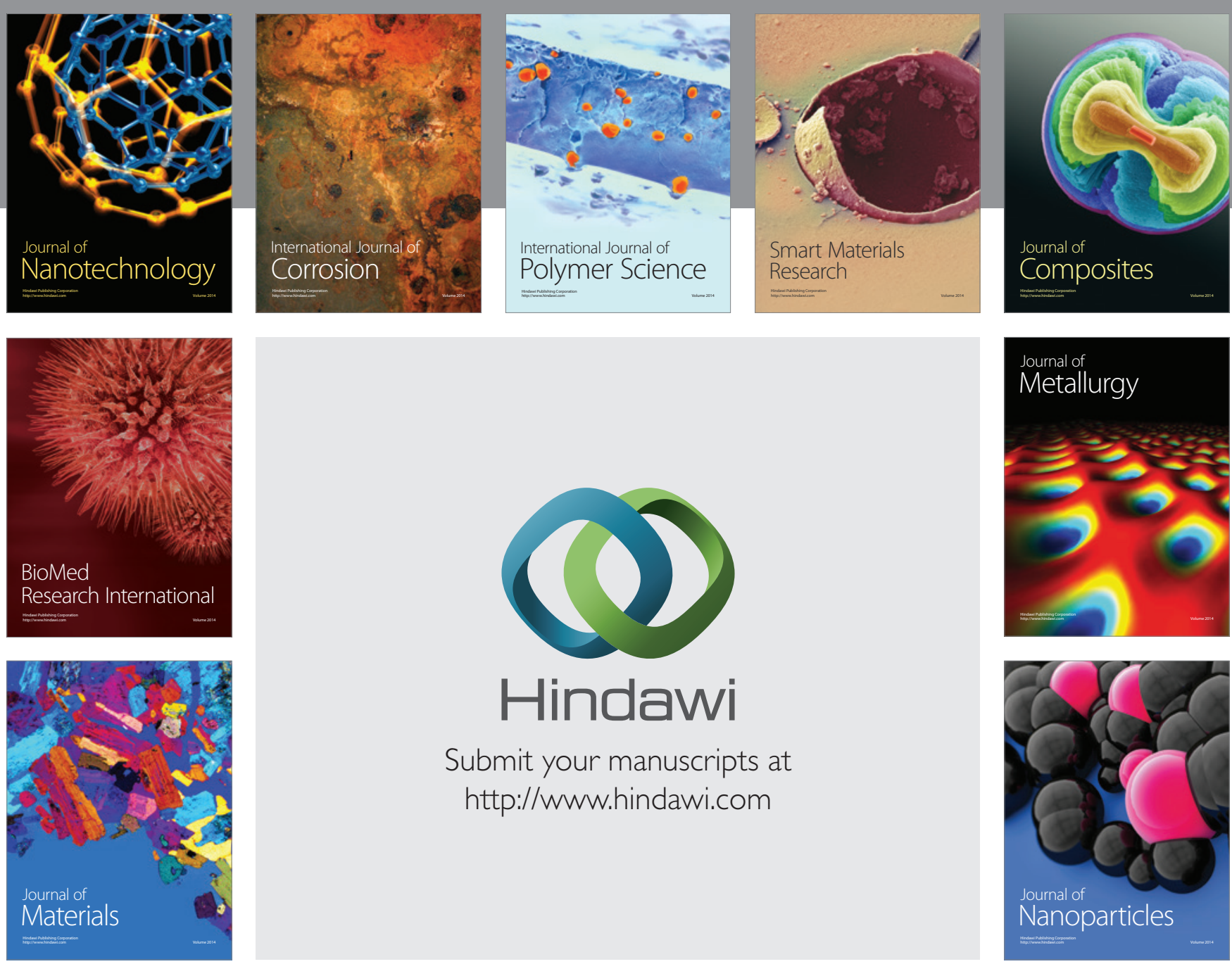

Submit your manuscripts at http://www.hindawi.com
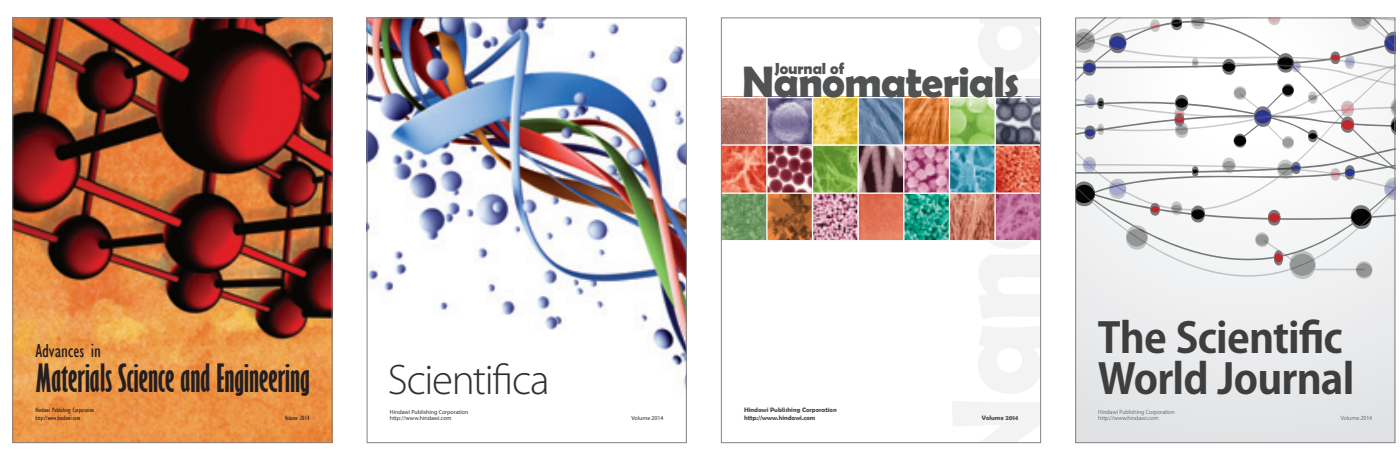

\section{The Scientific World Journal}
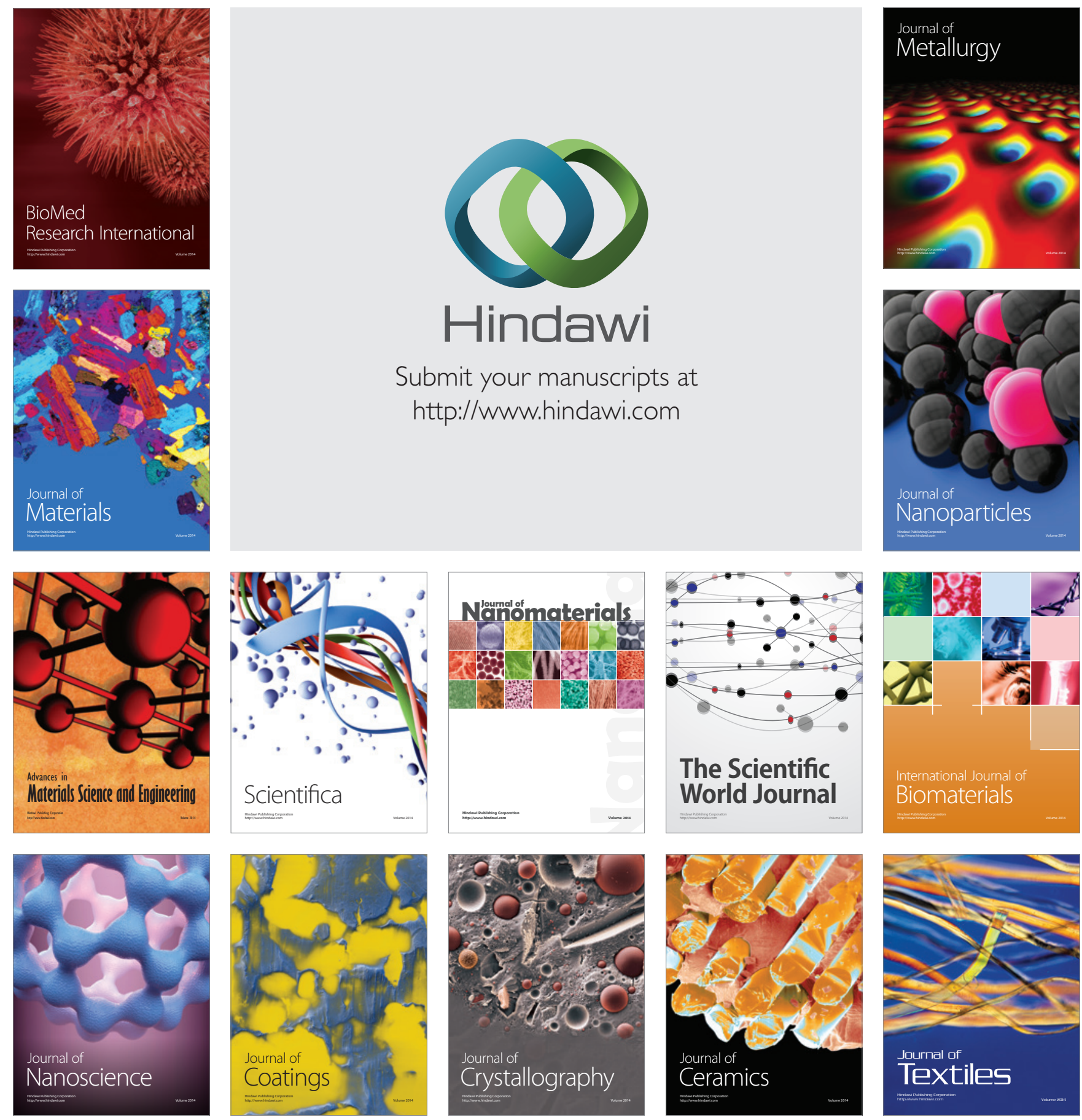